\title{
A Simple Sirs Mathematical Model with Mass Action Type Incidence
}

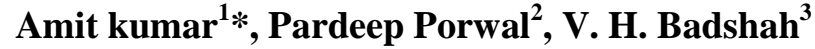 \\ ${ }^{1 *}$ School of Studies in Mathematics, Vikram University, Ujjain, India \\ ${ }^{2}$ School of Studies in Mathematics, Vikram University, Ujjain, India \\ ${ }^{3}$ School of Studies in Mathematics, Vikram University, Ujjain, India \\ *Corresponding Author: amit0830@gmail.com, Tel.: +91-9039709499
}

Available online at: www.isroset.org

Received $12^{\text {th }}$ Sep 2017, Revised $26^{\text {th }}$ Sep 2017 , Accepted $17^{\text {th }}$ Oct 2017 , Online $30^{\text {th }}$ Oct 2017

\begin{abstract}
In this paper a simple SIRS mathematical model with mass action type incidence is formulated and studied. Steady state, equilibrium point and the basic reproduction number are obtained for the system of differential equation. Existence and stability of the diseases free and endemic stages are investigated. An example is also furnished which demonstrates validity of main result.
\end{abstract}

Keywords - Mathematical Model, Equilibrium point, Stability, Incidence.

\section{INTRODUCTION}

Mathematical modeling is an essential tool to know and predict the spread of communicable diseases. In this progression, rate of incidence plays a important role. The incidence in a mathematical model is pace at which susceptible become communicable. The first SIR epidemic model was studied by Kermack and Mc Kendric [16] in the year 1927. Mena Lorca and Hethcote [6] also analyzed. The SIRS epidemic model has been studied by various authors viz. Capaso and Serio [15], Porwal and Badshah [7,8,9,10,11], Hethcote [2,3,4], Anderson and May [12], Kumar et al. [9] studied modified SIRS epidemic model with immigration and saturated incidence rate.

Communicable diseases create a constant threat to human beings. Each person on the earth can be affected by a disease. The emergence and re-emergence of infectious diseases have become a noteworthy universal problem. Accurate understanding of transmission mechanisms of diseases caused by existing and new pathogens may facilitate devising prevention tools. Prevention tools against transmissions, including vaccines and drugs, need to be developed at a similar pace to that of the microbes. Implementation and proper use of these sophisticated tools against the microbes is one more challenge.

In this paper, we extend the model of Hethcote [5] by taking SIRS mathematical model with mass action type incidence in place of SIR mathematical model. Further we study the model and obtain diseases free and endemic equilibrium of the system and analyze for stability. Also give an example for verification of our results.

\section{The MAThematical Model}

The proposed model describes a simple SIRS mathematical model with mass action type incidence. Here we adopt the following SIRS model:

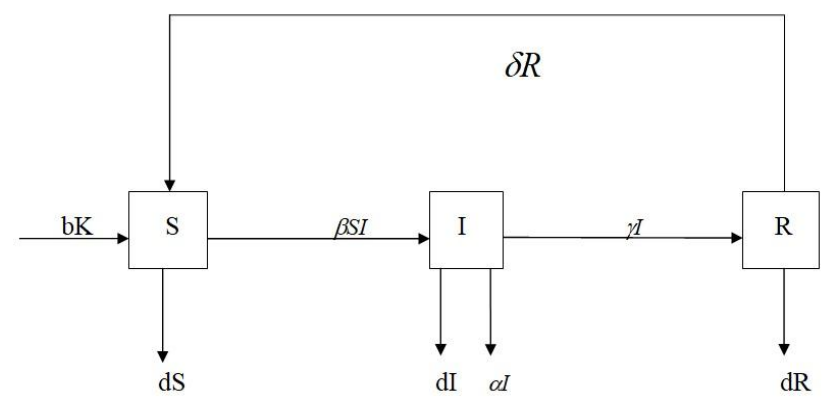

$$
\left.\begin{array}{l}
\frac{d S}{d t}=b K-\beta S I-d S+\delta R \\
\frac{d I}{d t}=\beta S I-(\gamma+\alpha+d) I \\
\frac{d R}{d t}=\gamma I-(\delta+d) R \\
\frac{d N}{d t}=b K-d N-\alpha I
\end{array}\right\}
$$

where $N=S+I+R$

and $S=$ Number of Susceptible,

$\mathrm{I}=$ Number of Infectious,

$\mathrm{R}=$ Recovered Compartment,

$\mathrm{N}=$ Total Population Size

With other Parameters in the model are

$\mathrm{b}=$ Birth Rate, 
$\mathrm{K}=$ Number of individual in the population

$\mathrm{d}=$ Natural Death Rate,

$\alpha=$ Disease Induced Death Rate,

$\beta=$ Transmission Coefficient,

$\delta=$ Loss of Immunity Rate Constant,

$\gamma=$ Recovery Rate,

\section{STEAdY STATE}

The disease free equilibrium of the model (2.1) is obtained by setting right hand side of model (2.1) equals to zero and taking $I=0$, we get.

$$
\left.\begin{array}{l}
b K-\beta S I-d S+\delta R=0 \\
\beta S I-(\gamma+\alpha+d) I=0 \\
\gamma I-(\delta+d) R=0 \\
b K-d N-\alpha I=0
\end{array}\right\}
$$

From the third equation of system (3.1), we get

$$
\begin{aligned}
& 0-(\delta+d) R=0 \\
& R=0 .
\end{aligned}
$$

and from first equation of system (3.1), we get

$$
\begin{aligned}
& b K-0-d S+0=0 \\
& S=\frac{b K}{d} .
\end{aligned}
$$

Hence, the disease free equilibrium point $E_{0}$ is

$$
E_{0}(S, I, R)=E_{0}\left(\frac{b K}{d}, 0,0\right) .
$$

From Second equation of system (3.1)

$$
\begin{aligned}
& \beta S-(\gamma+\alpha+d)=0 \\
& S^{*}=\frac{(\gamma+\alpha+d)}{\beta}
\end{aligned}
$$

by third equation of system (3.1)

$$
\begin{aligned}
& \gamma-(\delta+d) R=0 \\
& R^{*}=\frac{\gamma^{*}}{\delta+d}
\end{aligned}
$$

Again by first equation of system (3.1) we get

$$
\begin{gathered}
b K-\beta S^{*} I-d S^{*}+\delta R^{*}=0, \\
b K-d S^{*}=\left(\gamma+\alpha+d-\frac{\delta \gamma}{\delta+d}\right) I^{*},
\end{gathered}
$$

where

$$
I^{*}=\frac{b K-d S^{*}}{\left(\gamma+\alpha+d-\frac{\delta \gamma}{\delta+d}\right)}
$$

$$
I^{*}=\frac{b \beta K-d(\gamma+\alpha+d)}{\beta\left(\gamma+\alpha+d-\frac{\delta \gamma}{\delta+d}\right)} .
$$

Hence endemic equilibrium point is $E_{1}\left(S^{*}, I^{*}, R^{*}\right)$,

where

$$
\begin{gathered}
S^{*}=\frac{(\gamma+\alpha+d)}{\beta}, \\
I^{*}=\frac{b \beta K-d(\gamma+\alpha+d)}{\beta\left(\gamma+\alpha+d-\frac{\delta \gamma}{\delta+d}\right)}, \\
R^{*}=\frac{\gamma I^{*}}{\delta+d}
\end{gathered}
$$

The Basic Reproduction number

$$
R_{0}=\frac{b \beta K}{d(\gamma+\alpha+d)}
$$

\section{STABILITY ANALYSIS}

To discuss the stability of the model (2.1) the governing dynamical system is

$$
\left.\begin{array}{l}
F_{1}=b K-\beta S I-d S+\delta R \\
F_{2}=\beta S I-(\gamma+\alpha+d) I \\
F_{3}=\gamma I-(\delta+d) R
\end{array}\right\} .
$$

The Jacobian matrix of system (4.1) is

$$
J=\left[\begin{array}{lll}
\frac{\partial F_{1}}{\partial S} & \frac{\partial F_{1}}{\partial I} & \frac{\partial F_{1}}{\partial R} \\
\frac{\partial F_{2}}{\partial S} & \frac{\partial F_{2}}{\partial I} & \frac{\partial F_{2}}{\partial R} \\
\frac{\partial F_{3}}{\partial S} & \frac{\partial F_{3}}{\partial I} & \frac{\partial F_{3}}{\partial R}
\end{array}\right]
$$

$$
=\left[\begin{array}{lcr}
-\beta I-d & -\beta S & \delta \\
\beta I & \beta S-(\gamma+\alpha+d) & 0 \\
0 & \gamma & -(\delta+d)
\end{array}\right] .
$$

At equilibrium point $E_{0}$, the Jacobian matrix is 
$J_{E_{0}}=\left[\begin{array}{ccc}-d & \frac{-\beta b K}{d} & \delta \\ 0 & \frac{\beta b K}{d}-(\gamma+\alpha+d) & 0 \\ 0 & \gamma & -(\delta+d)\end{array}\right]$.

Its characteristics equation is

$$
\left|J_{E_{0}}-\lambda I\right|=0
$$

where

$$
J_{E_{0}}=\left[\begin{array}{ccc}
-d-\lambda & -\frac{\beta b K}{d} & \delta \\
0 & \frac{\beta b K}{d}-(\gamma+\alpha+d)-\lambda & 0 \\
0 & \gamma
\end{array}\right]=0,
$$

Whose eigen values are

$$
\lambda_{1}=-d, \lambda_{2}=\frac{\beta b K}{d}-(\gamma+\alpha+d)=R_{0}-1
$$

and $\lambda_{3}=-(\delta+d)$.

All the eigen values are negative if $\lambda_{2}<0$

$$
\begin{gathered}
\frac{\beta b K}{d}-(\gamma+\alpha+d)<0, \\
\frac{\beta b K}{d}<(\gamma+\alpha+d), \\
\frac{\beta b K}{d(\gamma+\alpha+d)}<1 \\
R_{0}<1,
\end{gathered}
$$

where

$$
R_{0}=\frac{b \beta K}{d(\gamma+\alpha+d)} \text {. }
$$

Hence the equilibrium point $E_{0}$ is locally asymptotically stable if $R_{0}<1$ and unstable if $R_{0}>1$. It is also globally asymptotically stable if $R_{0}<1$.

At equilibrium point $E_{1}$ the Jacobian matrix is

$$
J_{E_{1}}=\left[\begin{array}{ccc}
-\beta I^{*}-d & -\beta S^{*} & \delta \\
\beta I^{*} & \beta S^{*}-(\gamma+\alpha+d) & 0 \\
0 & \gamma & -(\delta+d)
\end{array}\right] .
$$

Its characteristics equation

$$
\left|J_{E_{1}}-\rho I\right|=0
$$

$$
\begin{aligned}
& =\left[\begin{array}{ccc}
-\beta I^{*}-d-\rho & -\beta S^{*} & \delta \\
\beta I^{*} & \beta S^{*}-(\gamma+\alpha+d)-\rho & 0 \\
0 & \gamma & -(\delta+d)-\rho
\end{array}\right] \\
& =\left(\beta I^{*}-d-\rho\right)\left(\beta S^{*}-(\gamma+\alpha+d)-\rho\right)(-(\delta+d)-\rho) \\
& -\beta I^{*}\left(\beta S^{*}(\delta+d+\rho)-\gamma \delta\right)=0
\end{aligned}
$$

$$
\begin{gathered}
\Rightarrow\left(\beta I^{*}+d+\rho\right)\left(\beta S^{*}-(\gamma+\alpha+d)-\rho\right)(\delta+d+\rho) \\
\quad-\beta I^{*}\left(\beta S^{*}(\delta+d+\rho)-\gamma \delta\right)=0 \\
\Rightarrow-\rho^{3}-\rho^{2}\left[\beta\left(S^{*}+I^{*}\right)+\delta+d-\gamma-\alpha-2 d\right] \\
-\rho\left[\begin{array}{l}
\beta I^{*}(\gamma+\alpha+d)+d(\gamma+\alpha+d)+(\delta+d)(\gamma+\alpha+2 d) \\
+(\delta+d)\left(\beta S^{*}+\beta I^{*}\right)-d \beta S^{*}
\end{array}\right]
\end{gathered}
$$$$
\Rightarrow \rho^{3}+\rho^{2}\left[\beta\left(S^{*}+I^{*}\right)+\delta-(\gamma+\alpha+d)\right\rfloor
$$$$
+\rho\left[\begin{array}{l}
\left(\beta I^{*}+d\right)(\gamma+\alpha+d)+(\delta+d) \beta\left(S^{*}+I^{*}\right) \\
+(\gamma+\alpha+2 d)(\delta+d)-d \beta S^{*}
\end{array}\right]
$$$$
+\left[\begin{array}{l}
\left(\beta I^{*}+d\right)(\gamma+\alpha+d)(\delta+d) \\
-d \beta S^{*}(\delta+d)-\beta I^{*} \delta d
\end{array}\right]=0
$$

$\Rightarrow \rho^{3}+a_{1} \rho^{2}+a_{2} \rho+a_{3}=0$,

where

$$
\begin{gathered}
a_{1}=\beta\left(S^{*}+I^{*}\right)+\delta-(\gamma+\alpha+d), \\
a_{2}=\left(\beta I^{*}+d\right)(\gamma+\alpha+d)+\beta(\delta+d)\left(S^{*}+I^{*}\right) \\
\quad+(\delta+d)(\gamma+\alpha+2 d)-d \beta S^{*} \\
a_{3}=\left(\beta I^{*}+d\right)(\gamma+\alpha+d)(\delta+d) . \\
-d \beta S^{*}(\delta+d)-\beta I^{*} \gamma d
\end{gathered}
$$


Clearly

$a_{1}>0, a_{2}>0, a_{3}>0$ and $a_{1} a_{2}>a_{3}$ if $R_{0}>1$

and by the Routh Hurwitz Criteria , the endemic equilibrium is locally asymptotically stable for $R_{0}>1$.

\section{EXAMPLE}

We take the parameters of the system, $d=2.33$, $K=1.4, \quad b=2.9, \quad \delta=1, \quad \alpha=0.28, \quad \beta=1.2$, $\gamma=0.49$. Then $E_{0}=(1.7424,0,0)$ and $R_{0}=0.5<1$.

Hence the diseases free equilibrium is asymptotically stable.

Now, we take parameters of the system as $d=0.16, \quad K=1.4, \quad b=2.9, \quad \delta=1, \quad \alpha=0.5$, $\beta=1.2, \gamma=0.18$. Then $E_{1}=(0.7,5.764,0.894)$

and $R_{0}=4.905>1$. Hence the endemic equilibrium point $E_{1}$ is locally asymptotically stable.

Remark If $\delta=0$, then we gets one of the models of Hethcote [5].

\section{CONCLUSION}

In this paper, we see that the basic reproduction number plays an important role to control the disease. If $R_{0}<1$ then there is a disease free equilibrium which is locally stable, that is the disease dies out. But when $R_{0}>1$ then the diseases persist and the endemic equilibrium is stable.

\section{ACKNOWLEDGMENT (HEADING 5)}

I acknowledge my sincere thanks to Dr. Sandeep Tiwari School of Studies in Mathematics, Vikram University Ujjain for his kind co-operation in the extension of this research paper. I also extend my sincere thanks and gratitude to Dr. V. H. Badshah, Prof. and Head School of Studies in Mathematics Vikram University Ujjain for helping me in formulating and extracting new results.

\section{REFERENCES}

[1]. A. Kumar., P. Porwal, and V. H. Badshah., "Modified SIRS Epidemic Model with Immigration and Saturated Incidence rate", MAYFEB Journal of Mathematics ISSN,2371-6193,(4) , 7-12, 2016.

[2]. H. W. Hethcote., "Qualitative Analysis for Communicable Disease Models", Math., Bio science, 28, 335-356, 1976.

[3]. H. W. Hethcote., "Three Basic Epidemiological Models", In Applied Mathematical Ecology, L. Gross, T.G. Hallam and S.A. Levin, Springer, Berlin, 119-144, 1989.

[4]. H. W. Hethcote., and P. Driessch Van Den., "Some Epidemiological models with Non-linear Incidence". J. Math. Boil., 29, 271-287, 1991.

[5]. H. W. Hethchote., "The Mathematics of Infectious Disease", SIAM Review 42, $599-653,2000$.
[6]. J. M. Mena Lorca., and H. W. Hethcote., "Dynamic Models of Infectious Disease as Regulators of Population Sizes', Journal Math. Bio. 30, 693 - 716, 1992.

[7]. P. Porwal., P. Ausif., and S. K. Tiwari., “An SIS Model for Human and Bacterial Population with Modified Saturated Incidence Rate and Logistic Growth", International Journal of Modern Mathematical Science, 12(2), 98-111, 2011.

[8]. P. Porwal., and V. H. Badshah., "A Study of Avion Influenza (Birds- Flu) Epidemic with Vaccinated Human Population", International Journal of Mathematical Modeling Simulation and Application 5(12), 75 .

[9]. P. Porwal., and V. H. Badshah., "Modified Epidemic Model with Saturated Incidence rate and Reduced Transmission Under Treatment", International Journal of Mathematics Archieve, 4(12), 106-111, 2013.

[10].P. Porwal., and V. H. Badshah., "Dynamical study of SIRS Epidemic Model with Vaccinated Susceptibilit", Canadian Journal of Basic and Applied Science 2(4), 90-96, 2014.

[11]. P. Porwal., P. Shrivastava, and S. K. Tiwari., "Study of Single SIR Epidemic Model", Plegia Library Advance in Applied Science Research, 6(4),1- 4,2014.

[12].R. M. Anderson, and R.M. May., “ Population Biology of Infectious Disease", Nature, Springer Verlag, Berlin , Heidelberg, New York, 180, 361-367, 1979.

[13]. R. K. Miller., and AN. Michal., "Ordinary Differential equations" New York, Academic Press (1982)

[14]. V. H. Badshah., and A. Kumar., "A Study of Mathematical Modeling in Mathematics", International Journal of Scientific Research in Mathematical and Statistical Sciences, Vol.3, Issue.1, pp.1-3, 2016.

[15]. V. Capasso., and G. Serio., "A Generalization of the Kermack and Mc Kendrick Deterministic Epidemic Model", Math Bio science. 42, $41-61,1978$.

[16]. W. O. Kermack., and A. G. Mc Kendrick., "Contribution to the Mathematical Theory of Epidemics", Part1. Royal Society, London, 115, 700 - 721, 1927.

\section{AUTHORS PROFILE}

Dr. Viquar Hussain Badshah Prof \& Head School of Studies in Mathematics, Vikram University Ujjain (M.P.). He is a professor since 20 years and Professor \& Head School of Studies in Mathematics since last 8 years. He is working in many field of mathematics research field. He has more than 125 research papers published in national and international peer-reviewed reputed journals.

Dr. Pradeep Porwal was born in 1984 at Ujjain (M.P.), India. He obtained his Ph.d. degree under the supervision of renowned Mathematician Professor V. H. Badshah, Professor \& Head, School of Studies in Mathematics, Vikram University, Ujjain. He served Lokmanya Tilak Science and Commerce College, Ujjain and Joined School of Studies in Mathematics, Vikram University, Ujjain as a Guest Faculty in 2010. In the field of Mathematical Modeling (BioMathematics) he has more than 25 research papers published in national and international peer-reviewed reputed journals.

Mr. Amit Kumar currently working as a research scholar in School of Studies in Mathematics Vikram University Ujjain and trying to develope new idea in the field of Mathematical modeling since 2011. 